\title{
Advanced energy storage materials for building applications and their thermal performance characterization: a review
}

\begin{abstract}
Advanced energy storage technology based on phase change materials (PCMs) has received considerable attention over the last decade for used in various applications. Buildings are the major industry which needs this advanced technology to improve internal building comfort and the reduction of energy usage. However, the main barrier which affects the application of this technology in building sector is the method to incorporate the PCMs into the building materials and the method used to measure the effectiveness of the PCMs as TES in building. In this paper, a review on the TES systems based on PCMs, their thermo-physical and chemical properties, and potential application as TES for buildings have been carried out. The methodologies for the incorporation of PCMs into the building materials, and their thermal performance are discussed.
\end{abstract}

Keyword: Thermal energy storage; Phase change materials; Building; Energy saving; Temperature 